\title{
Unified Analysis of Cooperative Spectrum Sensing over Generalized Multipath Fading Channels
}

\author{
Lina Mohjazi ${ }^{1}$, Diana Dawoud ${ }^{1}$, Paschalis C. Sofotasios ${ }^{2,3}$, Sami Muhaidat ${ }^{1,4}$, Mehrdad Dianati $^{1}$, \\ Mikko Valkama ${ }^{2}$, and George K. Karagiannidis ${ }^{3,4}$ \\ ${ }^{1}$ Institute for Communication Systems, University of Surrey, GU2 7XH, Guildford, United Kingdom \\ e-mail: \{l.mohjazi; d.dawoud; m.dianati\}@surrey.ac.uk \\ ${ }^{2}$ Department of Electronics and Communications Engineering, Tampere University of Technology, 33101 Tampere, Finland \\ e-mail: \{paschalis.sofotasios; mikko.e.valkama\} @tut.fi \\ ${ }^{3}$ Department of Electrical and Computer Engineering, Aristotle University of Thessaloniki, 54124 Thessaloniki, Greece \\ e-mail: \{sofotasios; geokarag\}@auth.gr \\ ${ }^{4}$ Department of Electrical and Computer Engineering, Khalifa University, PO Box 127788, Abu Dhabi, United Arab Emirates \\ e-mail: muhaidat@ieee.org
}

\begin{abstract}
The present work is devoted to the analytic performance evaluation of cooperative spectrum sensing (CSS) over generalized fading channels. The proposed analysis is based on the efficient Gaussian-Finite-Mixture (GFM) that allows the derivation of a simple and accurate closed-form expression for the average probability of energy detection (ED) under different fading environments. Capitalizing on this, we derive generalized closed-form expressions for the global probabilities of detection for the CSS with two main hard centralized fusion rules, namely, the AND and the OR rules. The efficiency and usefulness of the proposed expressions is justified by comparing the corresponding complementary receiver operating characteristic (ROC) curves for both multipath and composite multipath/shadowing fading channels, which are otherwise particularly difficult to obtain. The offered analytic results are corroborated by respective results from computer simulations and it is shown that the corresponding performance depends significantly on both the severity of fading and the involved number of users in the collaborative network.
\end{abstract}

\section{INTRODUCTION}

The last decades witnessed a rapid growth of data-intensive multimedia and interactive services along with a widespread deployment of wireless communications in myriad applications. However, the remarkably high data rates and overall increased performance offered by such broadband services have been mainly accomplished at the expense of dramatically increased bandwidth requirements which lead to a significant scarcity of spectrum resources. In addition, the high variability of the traffic statistics across time, space, and frequency has rendered the utilization of the licensed spectrum, assigned by the rigid static spectrum allocation scheme, rather inefficient [1]. As a result, investigating alternative and more efficient spectrum management policies became inevitable and to this end, cognitive radio (CR) has been flagged as the most promising solution thanks to its capability to increase substantially the utilization of the available spectrum resources [2], [3].

The distinct improvement in spectral utilization, motivated by the advancement of CR technology, is based on allowing unlicensed users - also known as secondary users (SU) - to opportunistically access the spectrum allocated to the licensees - also known as primary users (PU) - when the latter are idle. As a result, it is essential that SUs are equipped with the capability to detect whether a frequency band is utilized by a PU or not. This procedure is crucial in enabling the opportunistic spectrum access paradigm, and is widely known as spectrum sensing (SS) [4]. Various SS methods have been proposed over the past years with energy detection (ED) constituting the most widely adopted detection method due to its blind structure and low implementation complexity [5]. The principle of operation of ED is based on the deployment of a radiometer where its output determines the corresponding decision by comparing the received energy level with a predefined energy threshold.

It is also recalled that the operation of cognitive radio networks (CRN) is highly dependent upon the severity of fading which imposes nontrivial challenges on a single SU. As a result, cooperative spectrum sensing (CSS) strategies have been shown to provide an effective solution to sensing errors and performance degradation due to the detrimental effects of fading phenomena and to overcome the hidden terminal problem, which is typically encountered due to shadowing effects [20]. The CSS is technically performed by reporting sensing detection results by individual SUs to a central unit, called the fusion center (FC). Importantly, this process is carried out following either the soft or hard combining schemes.

The detection performance of a CR system is typically evaluated on the basis of two criteria, namely the probability of detection, $P_{d}$, and the probability of false alarm, $P_{f}$. Furthermore, the underlying ED based SS is crucial to the performance of both primary and CR networks. In this context, it is useful to quantify the detrimental effects of fading on the detection performance [6]-[19] and the references therein. Based on this, several investigations have been devoted to analyzing the average probability of detection, $\overline{P_{d}}$, of unknown 
deterministic signals over various fading channels, such as Rayleigh, Rician, Weibull and Nakagami- $m$ fading channels [21], [22], [26], [27]. Likewise, the authors in [28], [29] analyzed ED over generalized fading conditions. However, in spite of the usefulness of these analyses, the derived expressions are typically complex and rather inconvenient, which raises the need for the derivation of more tractable algebraic representation [30]-[38] and the references therein. In addition, the authors in [20], [39]-[41] analyzed the performance over Rayleigh fading and shadowing conditions while the case of Nakagami- $m$ frequency-selective fading channels was recently evaluated in [23]. Moreover, a comparison on the impact of Rayleigh, Nakagami- $m$ and Ricean fading conditions on CSS was reported in [42].

Nevertheless, the aforementioned analyses assume fading conditions that are modeled by a single fading model, which is not a generic method since it limits the generality and usefulness of these expressions. Motivated by this, the present work is devoted to the derivation of an accurate and generalized closed-form approximation for the probability of detection in CSS that can be applied in the analysis of CR systems under different fading channels. This is realized with the aid of Gaussian-Finite-Mixture (GFM), which has been shown to provide accurate modeling of generalized fading condtions.

\section{LOCAL SPECTRUM SENSING Model}

We firstly revisit the methodology of sensing the presence of PU signals in the context of ED. It is recalled that ED is a blind scheme that does not require any priori information of the signal. Hence, the primary signal received at the $j^{\text {th }} \mathrm{SU}$, $s_{j}(t)$, is assumed to be an unknown deterministic signal while the signal received by the $\mathrm{SU}, r_{j}(t)$, can be formulated as the following binary hypothesis testing problem

$$
r_{j}(t)= \begin{cases}w_{j}(t) & H_{0_{j}} \\ h_{j} s_{j}(t)+w_{j}(t) & H_{1_{j}}\end{cases}
$$

where both $H_{0_{j}}$ and $H_{1_{j}}$ refer to the two hypotheses of signal absence and signal presence, respectively. Given that SUs are geographically distributed, it is reasonable to assume that they experience independent fading effects with channel gain $h_{j}=\alpha_{j} e^{j \theta_{j}}$ assumed to be constant during the period of observation. Also, $w_{j}(t)$ is modeled as additive white gaussian noise (AWGN) with zero mean and variance $\sigma^{2}$ while the noise samples are assumed to be independent and identically distributed (i.i.d) as well as independent of the signal samples.

During the observation process, the received signal at the $j^{\text {th }} \mathrm{SU}$ is firstly filtered and squared and then the energy of $N$ samples are combined to produce the local test statistic, $y_{j}$. Following that, the test statistic $y_{j}$ is compared to a predefined energy threshold, $\lambda$. It is assumed that $y_{j}$ is a sum of squares of $N$ Gaussian random variables (RVs), each with zero mean and unit variance under hypothesis $H_{0_{j}}$, and non-zero mean and unit variance under hypothesis $H_{1_{j}}$ [5]. To this effect, $y_{j}$ follows the central chi-square $X_{N}^{2}$ distribution with $N$ degrees of freedom under $H_{0_{j}}$ and the non-central chi-square $\tilde{X}_{N}^{2}$ distribution, under $H_{1_{j}}$, with $N$ degrees of freedom and noncentrality parameter $\zeta=2 \gamma_{j}$ where $\gamma_{j} \triangleq \alpha_{j}^{2} E_{s_{j}} / \sigma^{2}$ is the instantaneous signal-to-noise ratio (SNR) with $E_{s_{j}}$ denoting the signal energy over the observation period.

Based on the above, evaluating $y_{j}$ can lead to two critical performance measures, namely, the probability of false alarm, $P_{f}=P\left(y_{j}>\lambda \mid H_{0_{j}}\right)$, and the probability of missed detection, $P_{m d}=1-P_{d}$, where $P_{d}=P\left(y_{j}>\lambda \mid H_{1_{j}}\right)$, is the corresponding probability of detection. Therefore, the $P_{f}$ and $P_{d}$ measures over AWGN can be represented as [21]

$$
P_{f}=\frac{\Gamma\left(u, \frac{\lambda^{\prime}}{2}\right)}{\Gamma(u)}
$$

and

$$
P_{d}=Q_{u}\left(\sqrt{2 \gamma}, \sqrt{\lambda^{\prime}}\right)
$$

respectively, where $\lambda^{\prime}=\lambda / \sigma^{2}, \Gamma(.,$.$) is the upper incomplete$ gamma function [43] and $Q_{m}(.,$.$) is the generalized Marcum$ Q-function [44].

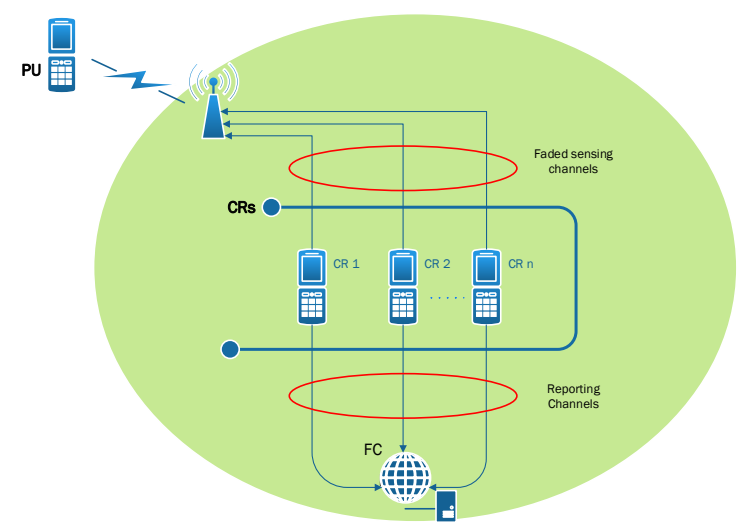

Fig. 1. Cooperative Spectrum Sensing System Model

\section{Spectrum Sensing over Fading Channels}

\section{A. Single User Energy Detection}

Evaluating the average $P_{d}$ over various fading channels requires averaging of the unconditional $P_{d}$ in (3) over the statistics of the corresponding fading process yielding

$$
\overline{P_{d}}=\int_{0}^{\infty} Q_{u}\left(\sqrt{2 \gamma}, \sqrt{\lambda^{\prime}}\right) f(\gamma) \mathrm{d} \gamma .
$$

It is recalled that the GFM approach constitutes a generic and rather useful distribution thanks to its capability to account for several fading environments. Its SNR PDF is given by

$$
p(\gamma) \simeq \frac{e^{-\frac{\mu_{i}^{2}}{2 \sigma_{i}^{2}}}}{\sqrt{2 \pi}} \sum_{i=1}^{G} \frac{\omega_{i}}{\bar{\gamma} \sigma_{i}} e^{-\frac{\gamma^{2}}{2 \bar{\gamma}^{2} \sigma_{i}^{2}}} e^{\frac{\gamma}{\gamma \sigma_{i}^{2}}}
$$

where $\bar{\gamma}=E\left[\alpha^{2}\right] E_{s} / \sigma^{2}$ denotes the corresponding average SNR, $G$ is the number of Gaussian components required to approximate $p(\gamma)$ within a predetermined accuracy whereas 
TABLE I

FM-EM PARAMETERS ESTIMATION OF SNR-PDF IN NAKAGAMI- $m$ FADING

\begin{tabular}{|l|l|l|l|}
\hline \hline$i$ & \multicolumn{1}{|c|}{$\omega_{i}$} & \multicolumn{1}{|c|}{$\mu_{i}$} & \multicolumn{1}{|c|}{$\sigma_{i}$} \\
\hline \hline 1 & 0.0078412 & 0.055048 & 0.023258 \\
\hline 2 & 0.028284 & 0.12687 & 0.042116 \\
\hline 3 & 0.065065 & 0.23212 & 0.067772 \\
\hline 4 & 0.12096 & 0.38377 & 0.10383 \\
\hline 5 & 0.17914 & 0.59372 & 0.15313 \\
\hline 6 & 0.21267 & 0.88112 & 0.22105 \\
\hline 7 & 0.19958 & 1.2726 & 0.31527 \\
\hline 8 & 0.12672 & 1.7983 & 0.44786 \\
\hline 9 & 0.051475 & 2.4981 & 0.65085 \\
\hline 10 & 0.0082675 & 3.4304 & 1.0519 \\
\hline
\end{tabular}

$\omega_{i}, \sigma_{i}^{2}$ and $\mu_{i}$ denote the weight, variance, and mean of the $i^{\text {th }}$ weighted PDF and are obtained by the well-known expectation maximization (EM) technique [45]. Thus, by substituting (5) into (4), the corresponding average $P_{d}$ is expressed as follows:

$$
\overline{P_{d}}=\sum_{i=1}^{G} \frac{\omega_{i} e^{-\frac{\mu_{i}^{2}}{2 \bar{\gamma} \sigma_{i}^{2}}}}{2 \pi \bar{\gamma} \sigma_{i}} \int_{0}^{\infty} Q_{u}\left(\sqrt{2 \gamma}, \sqrt{\lambda^{\prime}}\right) e^{-\frac{\gamma^{2}}{2 \bar{\gamma}^{2} \sigma_{i}^{2}}} e^{\frac{\gamma}{\bar{\gamma} \sigma_{i}^{2}}} \mathrm{~d} \gamma
$$

The $Q_{m}(a, b)$ can be also expressed according to [44], namely

$$
Q_{u}(\sqrt{2 \gamma}, \sqrt{\lambda})=1-\frac{\lambda^{\prime u-1} e^{-\frac{\gamma}{2}}}{2^{u-1} \Gamma(u)}\left(e^{\frac{\lambda^{\prime}}{2}}-1\right) .
$$

Therefore, by substituting (7) into (6) and recalling that

$$
Q(x) \triangleq \frac{1}{\sqrt{2 \pi}} \int_{x}^{\infty} e^{-\frac{y^{2}}{2}} \mathrm{~d} y
$$

it immediately follows that

$$
\begin{aligned}
\bar{P}_{d} \simeq & \overbrace{\sum_{i=1}^{G} \frac{\omega_{i}}{\sigma_{i} \bar{\gamma} \sqrt{2 \pi}} \int_{0}^{\infty} e^{-\frac{\left(\frac{\gamma}{\gamma}-\mu_{i}\right)^{2}}{2 \sigma_{i}^{2}}} \mathrm{~d} \gamma}^{T_{1}} \\
& -\underbrace{\left.\sum_{i=1}^{G} \frac{\omega_{i} \lambda^{\prime} u-1}{\sqrt{\pi} \sigma_{i} 2^{u-\frac{1}{2}} \bar{\gamma} \Gamma(u)} \int_{0}^{\frac{\lambda^{\prime}}{2}}-1\right)}_{T_{2}} e^{-\frac{\gamma}{2}-\frac{\left(\frac{\lambda^{\prime}}{\bar{\gamma}}-\mu_{i}\right)^{2}}{2 \sigma_{i}^{2}}} \mathrm{~d} \gamma
\end{aligned}
$$

Importantly, the above two terms can be expressed as

$$
T_{1}=\sum_{i=1}^{G} \omega_{i} Q\left(-\frac{\mu_{i}}{\sigma_{i}}\right)
$$

and

$$
T_{2}=\sum_{i=1}^{G} \omega_{i} B_{i} Q\left(\frac{\sigma_{i} \bar{\gamma}}{2}-\frac{\mu_{i}}{\sigma_{i}}\right)
$$

where

$$
B_{i}=\frac{\lambda^{\prime u-1}}{\Gamma(u) 2^{u-1}}\left(e^{\frac{\lambda^{\prime}}{2}}-1\right) e^{\frac{\sigma_{i}^{2} \bar{\gamma}^{2}}{8}-\mu_{i} \bar{\gamma}}
$$

To this effect, it immediately follows that the average prob-

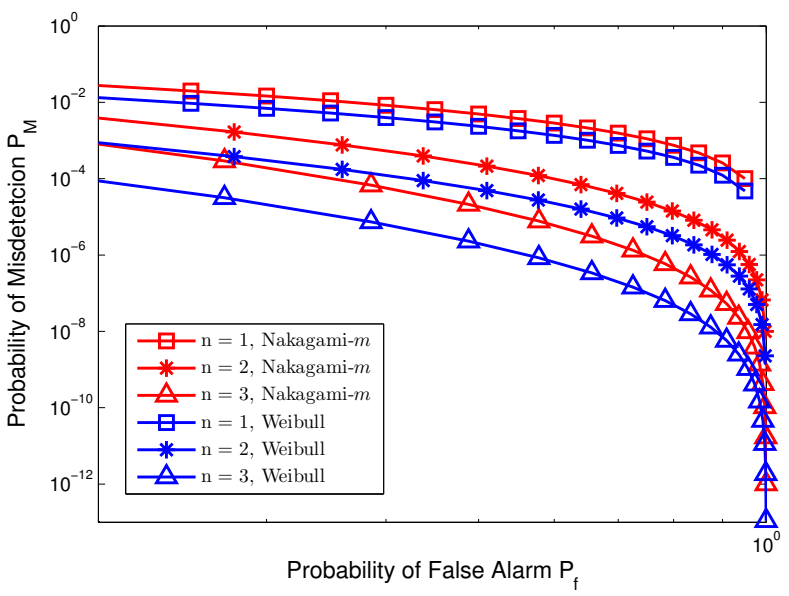

Fig. 2. Complimentary ROC curves of OR-rule under Weibull and Nakagami$m$ fading for 1,2 and 3 cooperative $\mathrm{CR}$ users $(\bar{\gamma}=10 \mathrm{~dB}, \mathrm{~N}=10, m=3$, and $\beta=4$ ).

ability of detection in the case of GFM can be expressed as

$$
\bar{P}_{d}=1-\sum_{i=1}^{G} \omega_{i} B_{i} Q\left(\frac{\sigma_{i} \bar{\gamma}}{2}-\frac{\mu_{i}}{\sigma_{i}}\right) .
$$

It is noted that the advantage of (13) is the relative simplicity to evaluate the $\overline{P_{d}}$ by using the weighting coefficients $\omega_{i}, \mu_{i}$, and $\sigma_{i}$, which also correspond to particular fading conditions.

\section{B. Cooperative Energy Detection}

As already mentioned, it has been shown extensively that cooperative SS increases substantially the corresponding probability of detection which is core principle in ensuring reliable and robust $\mathrm{CR}$ communications. The underlying CSS system model is illustrated in Fig. 1 where $n$ denotes the number of collaborating SUs experiencing fading conditions. Based on this, the global decision is made at the FC in order to determine the presence or absence of PU signals according to the following rule

$$
\widehat{H}=\sum_{j=1}^{n} D_{j} \begin{cases}<k & \widehat{H}_{0} \\ \geq k & \widehat{H}_{1}\end{cases}
$$

where $\widehat{H}$ is the sum of all locally sensed decisions from the collaborating SUs, denoted by $D_{j}$. However, although the soft decision may lead to a better sensing performance, the hard decision requires less signaling overhead, since the local sensing users only forward one-bit decision to the FC. Therefore, $D_{j}$ may take the values 0 or 1 . Furthermore, $\widehat{H}_{1}$ and $\widehat{H}_{0}$ denote the inferences drawn by the FC. The threshold $k$ is an integer, representing the " $k$-out-of- $n$ " voting rule. It can be seen in (14) that the AND-rule corresponds to the case of $k=n$ while the OR-rule corresponds to the case of $k=1$.

In order to evaluate the $\overline{P_{D}}$ and $P_{F}$ measures of CSS, we assume that all CRs use the same energy threshold, namely 
TABLE II

FM-EM PARAMETERS ESTIMATION OF SNR-PDF IN WEIBULL FADING

\begin{tabular}{|l|c|l|l|}
\hline \hline$i$ & \multicolumn{1}{|c|}{$\omega_{i}$} & \multicolumn{1}{|c|}{$\mu_{i}$} & \multicolumn{1}{|c|}{$\sigma_{i}$} \\
\hline \hline 1 & 0.0030069 & 0.047484 & 0.019959 \\
\hline 2 & 0.012766 & 0.11199 & 0.03721 \\
\hline 3 & 0.036897 & 0.20587 & 0.060056 \\
\hline 4 & 0.090249 & 0.34521 & 0.092605 \\
\hline 5 & 0.21692 & 0.54973 & 0.13579 \\
\hline 6 & 0.20854 & 0.80198 & 0.14918 \\
\hline 7 & 0.16376 & 1.0556 & 0.17694 \\
\hline 8 & 0.13681 & 1.2924 & 0.24035 \\
\hline 9 & 0.10815 & 1.541 & 0.33329 \\
\hline 10 & 0.022888 & 1.8733 & 0.43541 \\
\hline
\end{tabular}

$\lambda_{1}=\cdots=\lambda_{n}=\lambda$, which yields [20]

$$
{\overline{P_{D}}}_{A N D}=\prod_{j=1}^{n}\left\{\bar{P}_{d j}\left(\gamma_{j}\right)\right\}
$$

and

$$
P_{F, A N D}=\prod_{j=1}^{n}\left\{P_{f j}\left(\gamma_{j}\right)\right\}
$$

where $P_{d j}$ and $P_{f_{j}}$ are the individual probabilities of detection and false-alarm, respectively. On the contrary, the $\overline{P_{D}}$ and $P_{F}$ using an OR-rule based central CSS can be determined by

$$
\overline{P_{D}, O R}=1-\prod_{j=1}^{n}\left\{1-\bar{P}_{d j}\left(\gamma_{j}\right)\right\}
$$

and

$$
P_{F, O R}=1-\prod_{j=1}^{n}\left\{1-P_{f j}\left(\gamma_{j}\right)\right\} .
$$

To this effect, the $\overline{P_{D}}, A N D$ over generalized fading channels for the AND rule and CSS can be readily deduced by substituting (13) in (15) yielding

$$
{\overline{P_{D}, A N D}}=\prod_{j=1}^{n}\left\{1-\sum_{i=1}^{G} \omega_{i} B_{i} Q\left(\frac{\sigma_{i} \bar{\gamma}_{j}}{2}-\frac{\mu_{i}}{\sigma_{i}}\right)\right\} .
$$

Likewise, the $\overline{P_{D}}, O R$ in CSS can be obtained by substituting (13) in (17), resulting in

$$
{\overline{P_{D}, O R}}=1-\prod_{j=1}^{n}\left\{\sum_{i=1}^{G} \omega_{i} B_{i} Q\left(\frac{\sigma_{i} \bar{\gamma}_{j}}{2}-\frac{\mu_{i}}{\sigma_{i}}\right)\right\} .
$$

For both types of the central fusion rules, $P_{M}=1-P_{D}$.

\section{NumERICAL RESULTS}

The effectiveness of the derived unified probability expressions is demonstrated with the aid of complimentary ROC curves which are generated by simply estimating the weighting coefficients $\omega_{i}, \mu_{i}$, and $\sigma_{i}$ for the considered fading distributions. For example, the weights for the Nakagami- $m$ with $m=3$ and Weibull with $\beta=4$ models are depicted in Tables I and II, respectively, where $m$ and $\beta$ are parameters

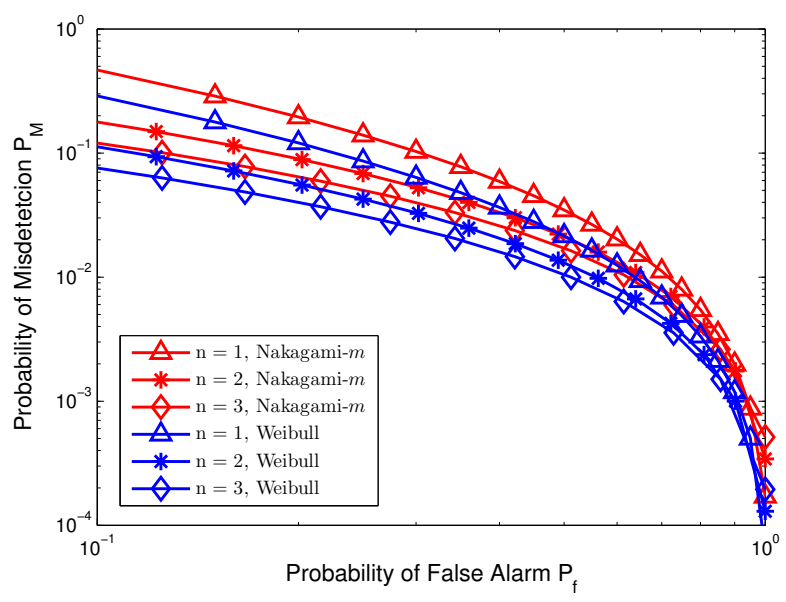

Fig. 3. Complimentary ROC curves of AND-rule under Weibull and Nakagami- $m$ fading for 1,2 and 3 cooperative CR users $(\bar{\gamma}=10 \mathrm{~dB}, \mathrm{~N}=10$, $m=3$, and $\beta=4$ ).

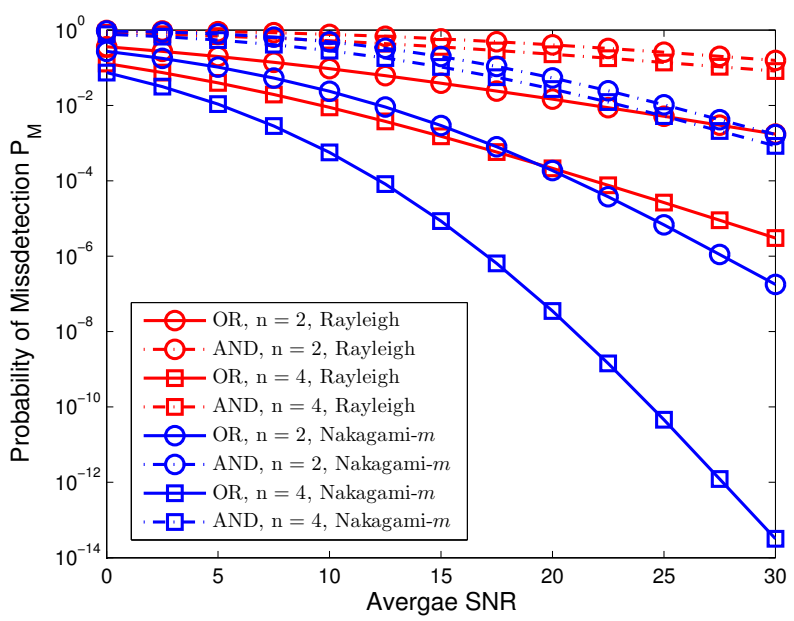

Fig. 4. $P_{M, O R}$ and $P_{M, A N D}$ vs. $\bar{\gamma}$ under Rayleigh and Nakagami- $m$ fading for 2 and 4 cooperative CR users $(\bar{\gamma}=10 \mathrm{~dB}, \mathrm{~N}=10, m=3)$.

relating to the severity of fading. These values were obtained with the aid of the EM algorithm with tolerance, $\varepsilon=10^{-6}$.

In the same context, Fig. 2 and Fig. 3 illustrate the ROC curves for the case of OR-rule and the AND-rule for a different number of cooperating users $n$. As expected, both figures show a significant improvement in the performance of the ED in $\mathrm{CSS}$ as the number of cooperative users increases. This can be readily observed by the degradation in $P_{M}$, compared to the single user case i.e., $n=1$. Furthermore, it can be clearly seen that the centralized CSS using OR-rule clearly outperforms the centralized CSS using AND-rule.

Fig. 4 depicts the probability of missed detection versus SNR to further illustrate the system performance by evaluating eq. (20) under Rayleigh and Nakagami- $m$ fading channels. As expected, the performance of the ED in CSS for both types of fading improves as the value of the average SNR increases. Moreover, the performance gain becomes much 


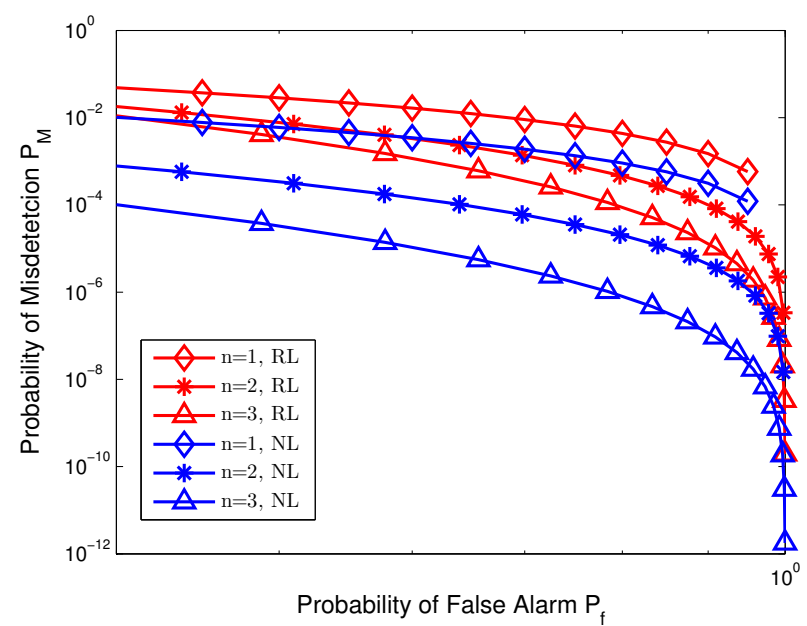

Fig. 5. Complimentary ROC curves of OR-rule under RL and NL composite fading for 1,2 and 3 cooperative CR users $(\bar{\gamma}=10 \mathrm{~dB}, \mathrm{~N}=10, m=3$, and $\zeta=1 \mathrm{~dB})$.

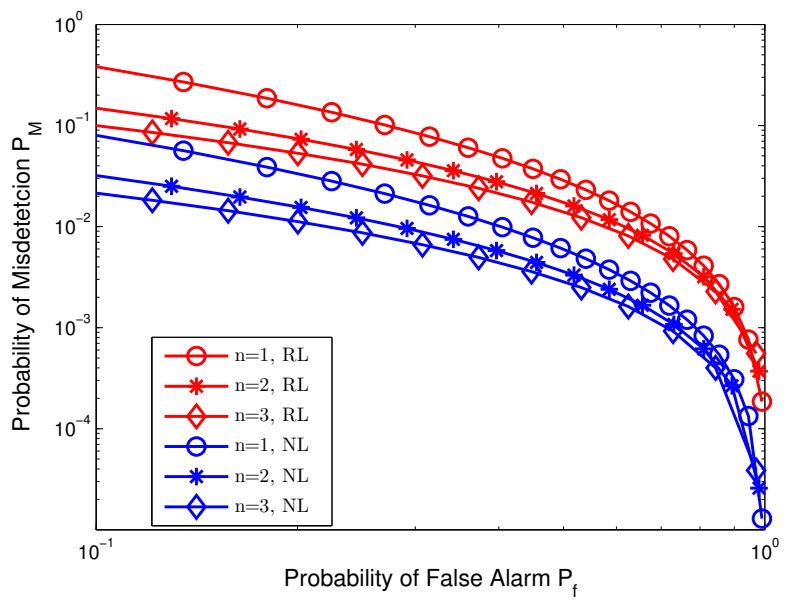

Fig. 6. Complimentary ROC curves of AND-rule under RL and NL composite fading for 1,2 and 3 cooperative CR users $(\bar{\gamma}=10 \mathrm{~dB}, \mathrm{~N}=10, m=3$, and $\zeta=1 \mathrm{~dB})$.

larger in the higher SNR regime as the number of cooperating users increases. It is also shown that for small values of $n$, all presented curves are almost parallel, indicating that they have the same diversity orders. However, for large values of $n$ the $P_{M}$ decreases slowly for low average SNR values and then decreases rapidly for high average SNR values, which implies a higher diversity order.

Furthermore, the performance of the proposed expression for CSS under composite multipath/shadowing fading channels is analyzed by means of complimentary ROC curves for the Rayleigh/Lognormal (RL) and Nakagami- $m / \operatorname{lognormal}(\mathrm{NL})$ fading conditions for the case of OR-rule and the AND-rule. These scenarios are presented in Fig. 5 and Fig. 6, respectively, for $\zeta$, that essentially defines the Lognormal distributions, set to $1 \mathrm{~dB}$. It is clearly observed that the performance of the CSS with the OR-rule outperforms its AND-rule counterpart.

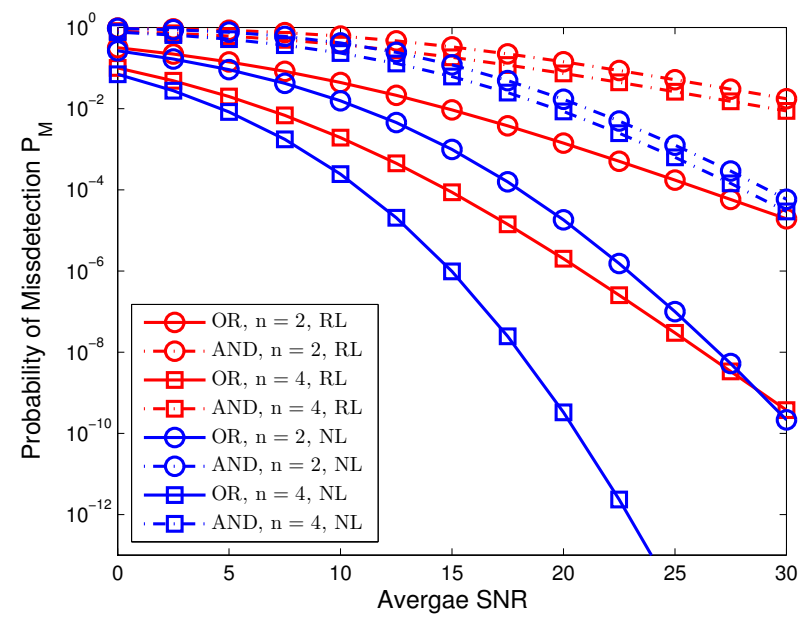

Fig. 7. $P_{M, O R}$ and $P_{M, A N D}$ vs. $\bar{\gamma}$ under RL and NL composite fading for 2 and 4 cooperative CR users $(\bar{\gamma}=10 \mathrm{~dB}, \mathrm{~N}=10, m=3$, and $\zeta=1 \mathrm{~dB})$.

Specifically, in the OR-rule, the performance improvement at increasing the number of cognitive users is more evident in the presence of non-severe fading conditions.

Finally, the derived expressions are used to study the effect of the average SNR on the performance of CSS. Indicatively, in the case of AND-rule, an increase of the number of sensing users do not significantly enhance the corresponding performance. On the contrary, the resulting performance is substantially improved as the number of sensing users increases, when employing the OR-rule. Also, the involved performance gain increases as the average SNR increases. Thus, it is evident that (20) and (19) are useful analytical tools in analyzing the performance of ED based SS over various fading conditions.

\section{CONCLUSions}

We provided an extensive analysis of energy detection spectrum sensing over generalized multipath fading channels. The corresponding probability of detection of cooperative spectrum sensing was determined by deriving unified expressions for the fusion center detection probabilities for both the ANDrule and the OR-rule strategies. The derived expressions are rather generic and relatively simple since they can account for various fading conditions. For example, they were employed in analyzing the spectrum sensing performance over Weibull and Nakagami- $m$ fading channels. Thus, they can be useful tools in future design and analysis of cognitive radio systems.

\section{ACKNOWLEDGMENTS}

This work was supported by the Finnish Funding Agency for Technology and Innovation (Tekes) under the project entitled "Energy-Efficient Wireless Networks and Connectivity of Devices-Systems (EWINE-S)", and by the Academy of Finland under the projects No. 251138 Digitally-Enhanced RF for Cognitive Radio Devices and No. 284694 "Fundamentals of Ultra Dense 5G Networks with Application to Machine Type Communication". 


\section{REFERENCES}

[1] Federal Communications Commission, "Spectrum policy task force," ET Docket, 02-135, Nov. 2003.

[2] S. Haykin, "Cognitive radio: brain-empowered wireless communications," IEEE J. Sel. Areas Commun., vol. 23, no. 2, pp. 201-220, Feb. 2005.

[3] J. Mitola, and Jr. G. Q. Maguire, "Cognitive radios: making software radios more personal," IEEE Pers. Commun., vol. 6, no. 4, pp. 13-18, Aug. 1999.

[4] T. Yucek, and H. Arslan, "A survey of spectrum sensing algorithms for cognitive radio applications," IEEE Commun. Surveys Tuts., vol. 11, no. 1, pp. 116-130, Jan. 2009.

[5] H. Urkowitz, "Energy detection of unknown deterministic signals," Proc. IEEE, vol. 55, no. 4, pp. 523-531, Apr. 1967.

[6] D. Zogas, G. K. Karagiannidis and S Kotsopoulos, "On the average output SNR in selection combining with three correlated branches over Nakagami $-m$ fading channels," IEEE Trans. Wireless Commun., vol. 3, no. 1, pp. 25-28, Jan. 2004.

[7] G. K Karagiannidis, D. Zogas, N.C Sagias, T. A. Tsiftsis, P. T. Mathiopoulos, "Multihop communications with fixed-gain relays over generalized fading channels," IEEE GLOBECOM '04, pp. 36-40.

[8] P. C. Sofotasios and S. Freear, "The $\kappa-\mu$ /gamma Composite Fading Model," IEEE ICWITS '10, Honolulu, HI, USA, Aug. 2010.

[9] K. Ho-Van, P. C. Sofotasios, and S. Freear, "Underlay Cooperative Cognitive Networks with Imperfect Nakagami- $m$ Fading Channel Information and Strict Transmit Power Constraint: Interference Statistics and Outage Probability Analysis," IEEE/KICS Journal of Communications and Networks, vol. 16, no. 1, pp. 10-17, Feb. 2014.

[10] D. Zogas and G. K. Karagiannidis, "Infinite-series representations associated with the bivariate Rician distribution and their applications," IEEE Trans. Commun., vol. 53, no. 11, pp. 1790-1794, Nov. 2005.

[11] P. C. Sofotasios and S. Freear, "The $\alpha-\kappa-\mu$ Extreme Distribution: Characterizing Non Linear Severe Fading Conditions," ATNAC '11, Melbourne, Australia, Nov. 2011

[12] D.S. Michalopoulos, G.K. Karagiannidis, T. A. Tsiftsis, and R. K. Mallik, "An optimized user selection method for cooperative diversity systems," IEEE GLOBECOM '06, pp. 1-6.

[13] P. C. Sofotasios, T. A. Tsiftsis, M. Ghogho, L. R. Wilhelmsson and M.Valkama, "The $\eta-\mu /$ Inverse-Gaussian Distribution: A Novel Physical Multipath /Shadowing Fading Model,” IEEE ICC '13, Budapest, Hungary, June 2013

[14] Z Hadzi-Velkov, N. Zlatanov, G.K. Karagiannidis "On the second order statistics of the multihop Rayleigh fading channel," IEEE Trans. Commun., vol. 57, no. 6, pp. 1815-1823, June 2009.

[15] P. C. Sofotasios, T. A. Tsiftsis, K. Ho-Van, S. Freear, L. R. Wilhelmsson, and M. Valkama, "The $\kappa-\mu /$ Inverse-Gaussian Composite Statistical Distribution in RF and FSO Wireless Channels," in IEEE VTC '13 Fall, Las Vegas, USA, pp. 1-5, Sep. 2013.

[16] S, Harput, P. C. Sofotasios, and S. Freear, "A Novel Composite Statistical Model For Ultrasound Applications," Proc. IEEE IUS '11, pp. 1-4, Orlando, FL, USA, 8-10 Oct. 2011.

[17] D. S Michalopoulos, A. S. Lioumpas, G. K. Karagiannidis and R. Schober "Selective cooperative relaying over time-varying channels," IEEE Trans. Commun., vol. 58, no. 8, pp. 2402-2412, Aug. 2010.

[18] P. C. Sofotasios, M. Valkama, Yu. A. Brychkov, T. A. Tsiftsis, S. Freear and G. K. Karagiannidis, "Analytic Solutions to a Marcum $Q-$ FunctionBased Integral and Application in Energy Detection," in CROWNCOM 14 Oulu, Finland, pp. 260-265, June 2014.

[19] P. C. Sofotasios, T. A. Tsiftsis, Yu. A. Brychkov, S. Freear, M. Valkama, and G. K. Karagiannidis, "Analytic Expressions and Bounds for Special Functions and Applications in Communication Theory," IEEE Trans. Inf. Theory, vol. 60, no. 12, pp. 7798-7823, Dec. 2014.

[20] A. Ghasemi, and E. S. Sousa, "Collaborative spectrum sensing for opportunistic access in fading environments," IEEE (DySPAN'05), Nov. 2005 , pp. $131-136$

[21] F. F. Digham, M. S. Alouini, and M. K. Simon, "On the energy detection of unknown signals over fading channels," IEEE Trans. Commun., vol. 55 , no. 1, pp. 21-24, Jan. 2007.

[22] O. Altrad, and S. Muhaidat, "A new mathematical analysis of the probability of detection in cognitive radio over fading channels," EURASIP J. on Wireless Commun. and Networking, no. 159, June 2013.
[23] Y. F. Sharkasi, D. McLernon, and M. Ghogho, "Cooperative spectrum sensing over frequency-selective Nakagami- $m$ fading channels," Sensor Signal Processing for Defence (SSPD'12), Sep. 2012, pp. 1-5.

[24] S. Kyperountas, N. Correal, S. Qicai, and Ye Zhuan, "Performance analysis of cooperative spectrum sensing in Suzuki fading channels," In Proc. CrownCom'07, Aug. 2007, pp. 428-432.

[25] A. Ghasemi, and E. S. Sousa, "Asymptotic performance of collaborative spectrum sensing under correlated Log-normal shadowing," IEEE Commun. Lett., vol. 11, no. 1, pp. 34-36, Jan. 2007.

[26] D. Horgan, and C. C. Murphy, "Fast and accurate approximations for the analysis of energy detection in Nakagami- $m$ channels," IEEE Commun Lett., vol. 17, no. 1, pp. 83-86, Jan. 2013.

[27] P. C. Sofotasios, M. K. Fikadu, H-V. Khuong, and M. Valkama, "Energy detection sensing of unknown signals over Weibull fading channels," In Proc. ATC '13, HoChiMinh City, Oct. 2013, pp. 414-419.

[28] Y. Fathi and M. H. Tawfik, "Versatile performance expression for energy detector over $\alpha-\mu$ generalized fading channels," IET Electronics Letters, vol. 48, no. 17, pp. 1081-1082, 2012.

[29] P. C. Sofotasios, E. Rebeiz, L. Zhang, T. A. Tsiftsis, D. Cabric, and S. Freear, "Energy detection based spectrum sensing over $\kappa-\mu$ and $\kappa-\mu$ extreme fading channels," IEEE Trans. Veh. Technol., vol. 62, no. 3, pp. 1031-1040, Mar. 2013

[30] P. C. Sofotasios, and S. Freear, "A novel representation for the Nuttall Q-function,” Proc. IEEE ICWITS '10, pp. 1-4, Hawaii, HI, USA, 28 Aug. - 3 Sep. 2010.

[31] P. C. Sofotasios, and S. Freear, "Novel expressions for the one and two dimensional Gaussian $Q$-functions," Proc. IEEE ICWITS '10, pp. 1-4, Hawaii, HI, USA, 28 Aug. -3 Sep. 2010.

[32] P. C. Sofotasios, and S. Freear, "Novel expressions for the Marcum and one dimensional $Q$-functions," Proc. ISWCS '10, pp. 736-740, York, UK, 18-21 Sep. 2010.

[33] P. C. Sofotasios, and S. Freear, "New analytic results for the incomplete Toronto function and incomplete Lipschitz-Hankel Integrals," Proc. IEEE VTC '11 Spring, pp. 1-4, Budapest, Hungary, 15-18 May 2011.

[34] P. C. Sofotasios, K. Ho-Van, T. D. Anh, and H. D. Quoc, "Analytic results for efficient computation of the Nuttall- $Q$ and incomplete Toronto functions," Proc. IEEE ATC '13, pp. 420-425, HoChiMinh City, Vietnam, 16-18 Oct. 2013

[35] P. C. Sofotasios, and S. Freear, "Simple and accurate approximations for the two dimensional Gaussian $Q$-Function," Proc. SBMO/IEEE IMOC '11, pp. 44-47, Natal, Brazil, 29-31 Oct. 2011.

[36] P. C. Sofotasios, and S. Freear, "Upper and lower bounds for the Rice Ie-function," IEEE ATNAC '11, pp. 1-4, Melbourne, Australia, 9-11 Nov. 2011

[37] P. C. Sofotasios, and S. Freear, "Analytic expressions for the Rice $I e-$ function and the incomplete Lipschitz-Hankel integrals," IEEE INDICON '11, pp. 1-6, Hyderabad, India, 16-18 Dec. 2011.

[38] P. C. Sofotasios, On Special Functions and Composite Statistical Distributions and Their Applications in Digital Communications over Fading Channels, Ph.D. Dissertation, University of Leeds, England, UK, 2010.

[39] A. Ghasemi, and E.S. Sousa, "Asymptotic performance of collaborative spectrum sensing under correlated log-normal shadowing," IEEE Commun. Lett., vol. 11, no. 1, pp. 34-36, Jan. 2007.

[40] S. Atapattu, C. Tellambura, and Hai Jiang, "Energy detection based cooperative spectrum sensing in cognitive radio networks," IEEE Trans. Wireless Commun., vol. 10, no. 4, pp. 1232-1241, Apr. 2011.

[41] Y.-J. Choi, W. Pak, Y. Xin, and S. Rangarajan, "Throughput analysis of cooperative spectrum sensing in Rayleigh-faded cognitive radio systems," IET Communications, vol. 6, no. 9, pp. 1104-1110, June 2012

[42] J. Duan, and Y. Li, "Performance analysis of cooperative spectrum sensing in different fading channels," Proc. IEEE ICCET '10, Apr. 2010.

[43] I. S. Gradshteyn, and I. M. Ryzhik, Table of Integrals, Series, and Products, Academic Press, $6^{\text {th }}$ Ed. San Diego, CA, 2000.

[44] S. Andras, A. Baricz, and Y. Sun, "The generalized Marcum Q-function: an orthogonal polynomial approach," Acta Universitatis Sapientiae Mathematica, vol. 3, no. 1, pp. 60-76, Jan. 2011

[45] S. Atapattu, C. Tellambura, and H. Jiang, "A mixture gamma distribution to model the SNR of wireless channels," IEEE Trans. Wireless Commun., vol. 10, no. 12, pp. 4193-4203, Dec. 2011. 